\title{
Testing the efficiency of abrasive treatment of reconditioned parts using coolant cleaning in a magnetic sump
}

\author{
Boris Avdeyev ${ }^{1, *}$, Aleksey Vyngra ${ }^{1}$ and Aleksander Bordiug ${ }^{1}$ \\ ${ }^{1}$ Kerch State Maritime Technological University, Ordzhonikidze st., 82, Kerch, 298309, Russia
}

\begin{abstract}
This article discusses pilot study of effectiveness of abrasive machining of the recoverable parts for cleaning cooling lubricants involved in technological processes of processing of the parts. One of the cleaning methods is a magnetic sump, which has high efficiency at relatively low cost of operation and maintenance. Laboratory tests of the magnetic sump were carried out according to a full-factor experiment, varying with certain parameters in order to identify the purity index of cooling lubricant when cleaning from mechanical impurities. Dependencies are obtained that connect the value of purity index of coolant with the technological and structural parameters of the magnetic sump. The influence of the cooling lubricant purity index in the magnetic sump on the roughness of grinded surface of the samples was revealed. The most rational parameters of the magnetic sump have been determined at which optimum surface roughness is observed when processing the engine crankshaft using cooling lubricant cleaned in the magnetic sump.
\end{abstract}

\section{Introduction}

When cutting, extruding, rolling, stamping, drilling parts, etc. often use cooling lubricants, which remove heat from the tool and workpiece and lubricate the rubbing parts. In the general case, use of cooling lubricant can increase intensity of technological processes, labour productivity and machine capacity, improve product quality [1,2]. During operation, cooling lubricant is contaminated with chips, solid dust, foreign oils or microorganisms. Pollution leads not only to decrease in efficiency and quality of processing of materials and, consequently, to their frequent renewal, but also to cause equipment breakdowns.

Use of a magnetic field to clean cooling lubricant is most effective when the number of magnetic particles allows their forced coagulation.

Main force acting on the particles in the sump is gravitational; except for it, when field is present, magnetic forces (attraction and coagulation) also act on particles [3]:

$$
F_{M}=\mu_{0} \chi V H \operatorname{Vrad} H,
$$

\footnotetext{
* Corresponding author: dirigeant@mail.ru
} 
where $\mu_{0}=$ const $=4 \pi \cdot 10^{-7}$ - magnetic constant, $\mathrm{H} / \mathrm{m} ; \chi$ - magnetic susceptibility, pu; $V$ particle volume, $\mathrm{m}^{3} ; H$, grad $H$ - strength of magnetic field and its gradient $\mathrm{A} / \mathrm{m} \mathrm{и} \mathrm{A} / \mathrm{m}^{2}$ respectively.

However, magnetic forces not only attract magnetic particles to zones with the highest field strength, but also serve to coagulate them [4]. Particles increase in size, under influence of magnetic (Coulomb) forces, forming of a needle-shaped floccules:

$$
F_{F}=\frac{4 \pi M_{1} M_{2}}{\mu_{0} \mu r^{2}},
$$

where $M_{1}, M_{2}$ - "magnetic masses" of the particles, $\mathrm{m}^{2} \cdot \mathrm{kg} / \mathrm{s}^{2} \cdot \mathrm{A} ; \mu$ - magnetic permeability of medium in which the particles are located; $\mathrm{r}$ - distance between particles, $\mathrm{m}$.

The magnetic field inside the working chamber increases not only the cleaning efficiency, but also reduces the mass dimensions, because coagulated particles have more weight, and accordingly, they require less time for sedimentation. Thus, volume of the sump and the residence time of the cooling lubricant in it are reduced [5,6].

Process of coagulation of the particles in a magnetic sump occurs directly in the working chamber $[7,8]$. Coagulated particles under influence of gravitational and magnetic forces settle on the poles of the electromagnetic system. The advantages of the sump include the factor that it captures soft magnetic impurities. This type of magnetic sumps is most widely used as it provides high cleaning efficiency at relatively low material and energy costs. Magnetic sumps often have baffles and non-magnetic nozzles $[9,10]$ in order to allow all contaminated flux to pass by magnetic field with a high strength.

\section{Objective}

Problem of identifying of processing efficiency of the material depending on cleaning parameters is acute. Based on it, the purpose of this article is to hold pilot study with the subsequent evaluation of results to determine the most rational parameters of the magnetic settling tank at which optimum surface roughness is observed when processing the engine crankshaft using coolant cleaned in a magnetic sump.

\section{The first stage of laboratory testing}

At the first stage, laboratory tests of the magnetic sump were carried out according to a fullfactor experiment (FFE), varying with certain parameters in order to determine purity index when cleaning from mechanical impurities.

Fig. 1 shows an installation diagram with which experimental studies were carried out to identify the effectiveness of cleaning of cooling lubricants from mechanical impurities in the magnetic sump. The unit includes a regulating transformer LATR 1 (laboratory autotransformer); voltmeter 2; ammeter 3; block of rectifier diodes 4; magnetic sump 5 .

The tests were carried out according to generally accepted technique with the following variable parameters: current strength in the winding of electromagnet $I=5 ; 10 ; 15 ; 20 ; 25$; 30 A. A $3 \%$ emulsion Ukrinol -1 with concentrations of solids $\delta_{\text {In }}=0,25 ; 0,7 ; 1,0$ g/l was used as a cooling lubricant. For the values of the input concentration $\delta_{I n}$, we took the average size distributions of impurities in cooling lubricants during the grinding of reconditioned crankshafts of ZIL engines. The rate of horizontal motion of the cooling lubricant $v_{n}$ at the beginning of the sump was taken $9-12 \mathrm{~mm} / \mathrm{s}$. The limiting value of purity index $\delta_{\text {Out }}$ of cooling lubricant during operation is taken equal to $\delta_{\text {Out }}=0,1 \mathrm{~g} / \mathrm{l}$. 


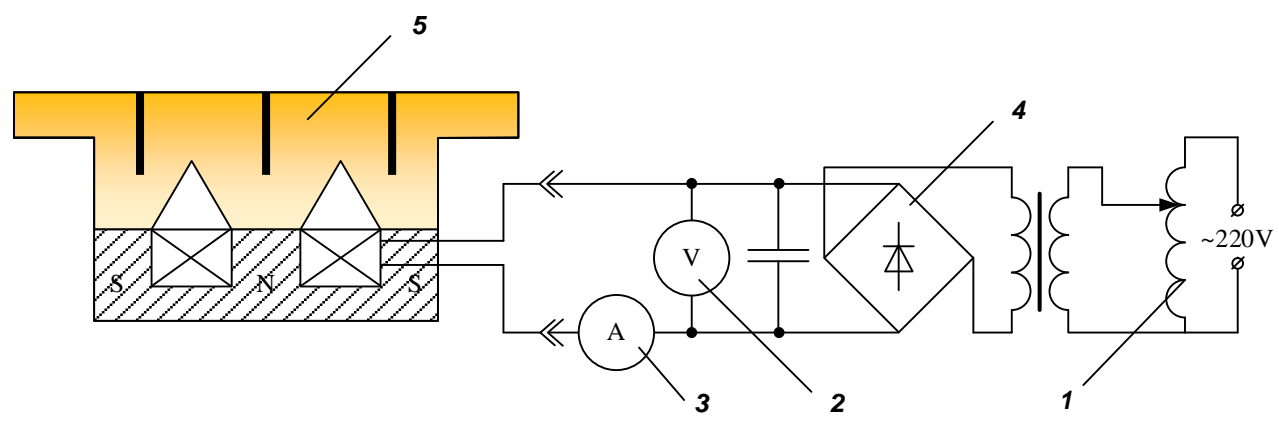

Fig. 1. Diagram and photo of an experimental unit for determining of effectiveness of cleaning of cooling lubricant from mechanical impurities in a magnetic sump

At the first stage, studies were carried out with the number of turns in the electromagnet winding equal to 2800. In this case, cooling lubricant with a concentration of mechanical impurities $\delta_{\text {In }}$ equal to $1,0 \mathrm{~g} / \mathrm{l}$ was fed into the sump. In addition, magnitude of current $I$ was changed in the winding of the electromagnet. Moreover, after each change in amperage, samples of purified cooling lubricant were taken and the mass concentration of mechanical impurities was determined according to the technique [11].

According to the results of the experiment, relationship was obtained that relates the value of purity of cooling lubricant $\delta_{\text {Out }}$ to the technological and structural parameters of the magnetic sump $\delta_{\text {Out }}=f\left(I, \delta_{\text {In }}\right)$ (Fig. 2). The calculation here and hereinafter was carried out according to the procedure presented in [11].

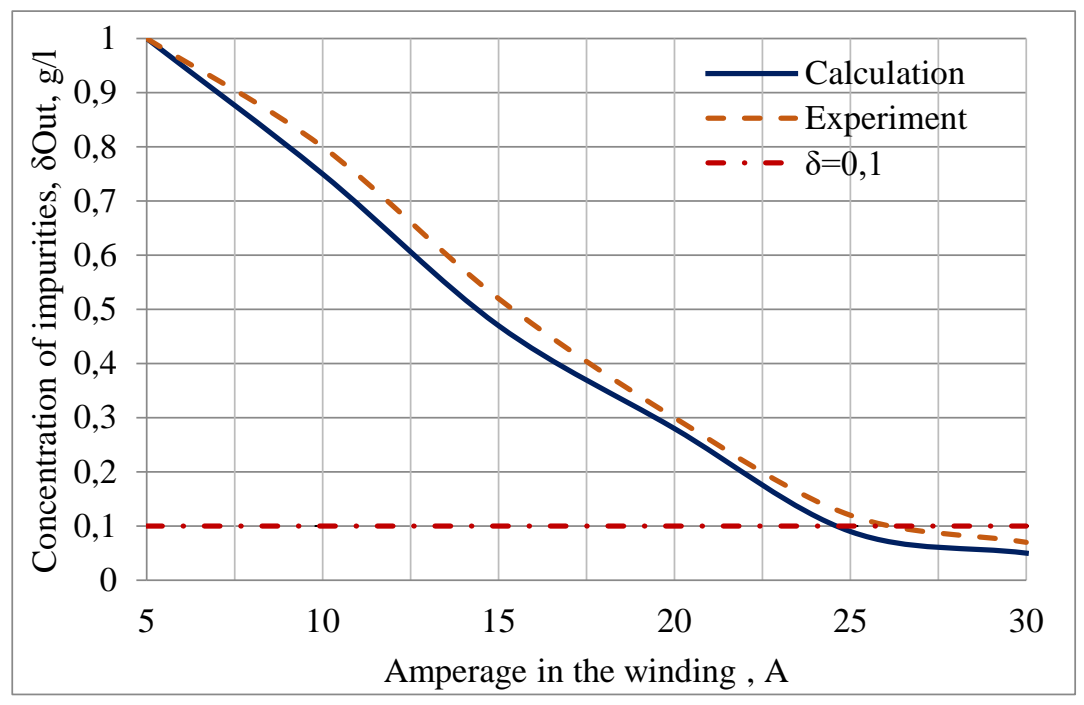

Fig. 2. The dependence of the purity of cooling lubricant on the current change in the winding at $\delta_{\text {In }}=$ $1 \mathrm{~g} / \mathrm{l}$

Technique of the above mentioned experiment was implemented for the following designated concentrations: $\delta_{\text {In }}=0,7 \mathrm{~g} / 1$ и $\delta_{\text {In }}=0,25 \mathrm{~g} / \mathrm{l}$. The results of experiments are presented in Fig. 3, 4. 


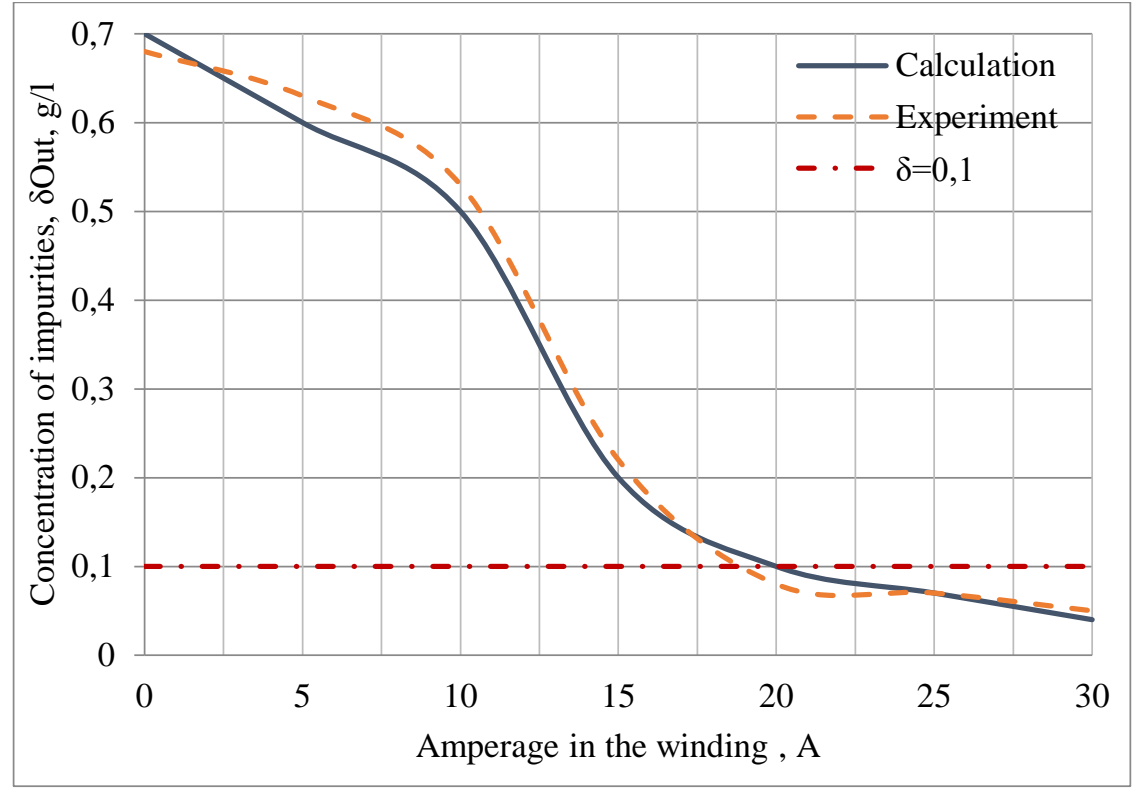

Fig. 3. Dependence of purity index of cooling lubricant on current change in the winding at $\delta_{\text {In }}=0,7$ $\mathrm{g} / \mathrm{l}$

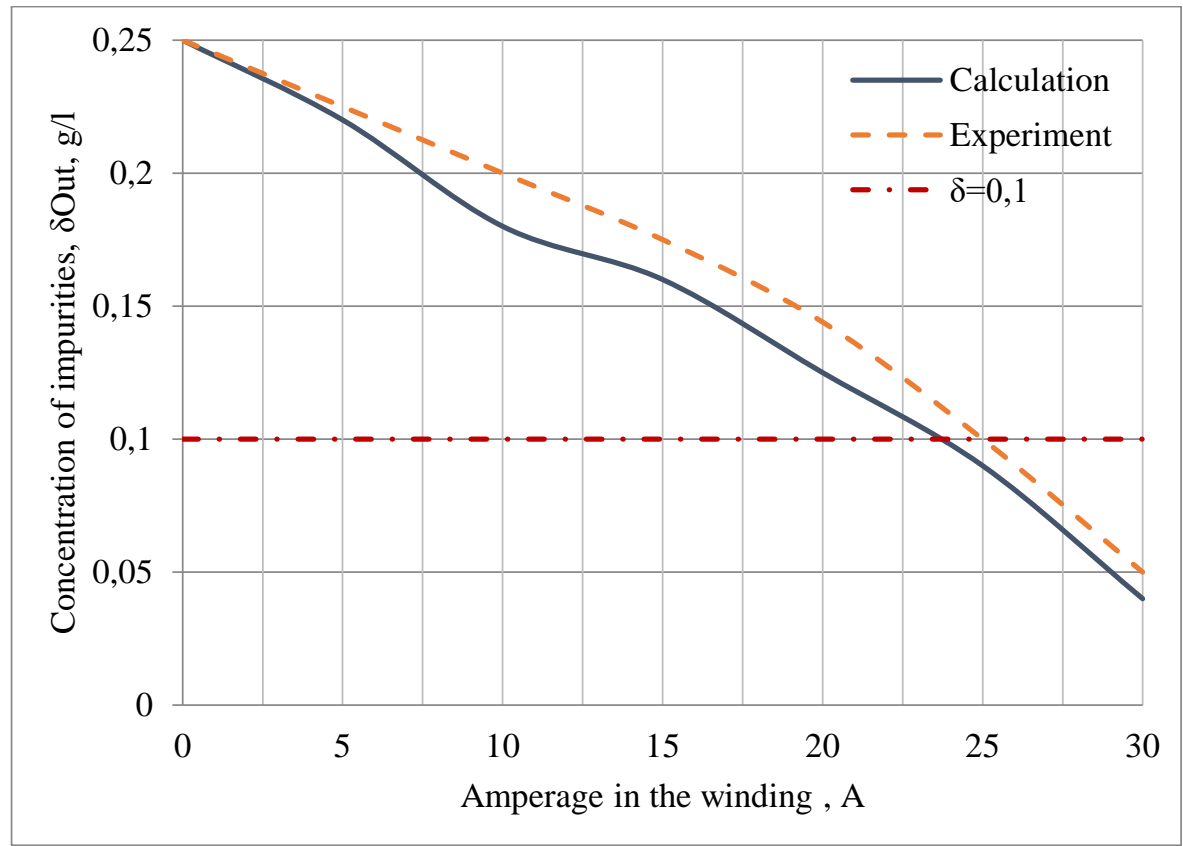

Fig. 4. Dependence of the purity index of cooling lubricant on current change in the winding at $\delta_{\text {In }}=$ $0,25 \mathrm{~g} / \mathrm{l}$

The effectiveness of cleaning of cooling lubricant from the magnetic field strength in the sump is of particular practical interest. This dependence $(\eta=f(H))$ is presented in Fig. 5 


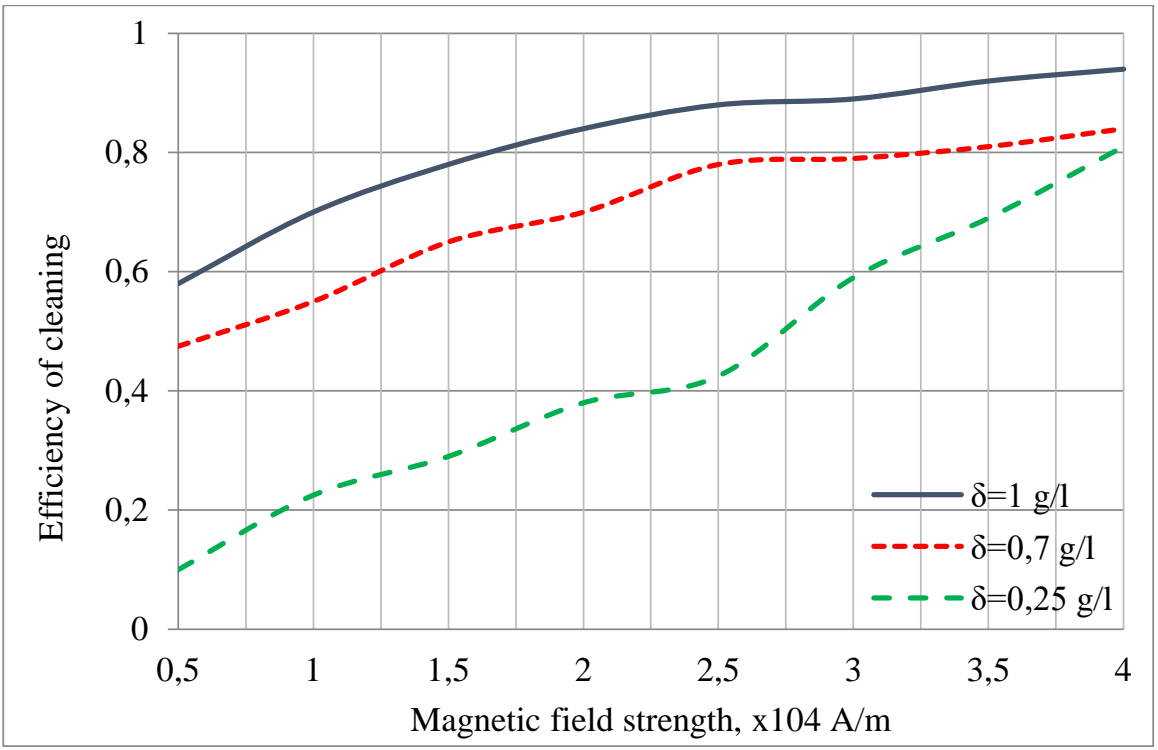

Fig. 5. Dependence of the efficiency of cleaning of the cooling lubricant on the magnetic field in the sump

The errors of the experimental and calculated dependencies did not exceed $10 \%$.

\section{The second stage of laboratory testing}

The second stage of laboratory testing of effectiveness of abrasive processing of the reconditioned parts using cleaning of cooling lubricant in a magnetic sump were carried out at the second stage of the research.

The reason for pilot study was the need to increase productivity of machining [12]:

a) by increasing durability of the grinding wheel and a corresponding reduction in the number of dressings;

b) by intensification of grinding modes (reduction of machine time);

c) reducing the consumption of grinding wheels and diamond ruling tools.

Moreover, in all cases, a prerequisite is to ensure the required quality of grinded parts according to State All-Russian Standard 2789-73. Conditions of abrasive processing of the reconditioned parts require use of not only different grades of grinding materials, but also certain sizes (graininess) of the base grit (abrasive grain - $160 \ldots 2000 \mu \mathrm{m}$; abrasive powders - $40 \ldots 125 \mu \mathrm{m}$ ), as well as a variety of types of cooling lubricants used in the processing of parts.

In this regard, the studies were carried out on a OD grinder - model 3A151, with electrocorundum grinding wheels with a graininess of $40-100 \mu \mathrm{m}$ and a hardness of CT1 and a size of 1 (ПП) $900 \times 50 \times 305$, according to the requirements of State All-Russian Standard 2424-83, samples of welded shaft journals of a ZIL automobile from steel 45 State AllRussian Standard 1050-88 with a hardness of 50-60 HRC. Cooling lubricant (2\% emulsion Ukrinol - 1- a mixture of mineral oil, emulsifiers, and corrosion inhibitors) was fed as a free falling jet (watering), the flow rate of which was 10-12 l/min. The dressing of circle was carried out with a diamond in the frame (type 037-3908, 1037-0.04-64). - The size of the machining allowances and the recommended grinding modes were determined by the analytical calculation method [24]. The roughness parameters of the processed surfaces were measured using a profilograph — model 201 profilometer and visual analysis of the profile 
on the metallurgical microscope MIS 11 [13]. The presence of a thermal damage on the grinded surface and the assessment of the condition of the circle surface were carried out visually using a MIM-6 microscope equipped with a digital camera.

At the beginning of the experimental research, preliminary grinding (rough grinding) ( $t_{p}$ $=0,03 \mathrm{~mm}$ ) of the samples after hardfacing was carried out, providing a surface roughness $R_{a}=1,6 \mu \mathrm{m}$. Next, the final grinding (finishing) was performed ( $t_{p}=0,005 \mathrm{~mm}$ ). The required parameter of the surface roughness of the component was $R_{a}=0,32 \mu \mathrm{m}$.

The concentration of mechanical impurities in the cooling lubricant $\delta_{I n}$, increases with long-term grinding and removal of large non-uniform allowances, thereby deteriorating the roughness parameter.

In addition, the frequency of cleaning of the grinding wheel increases, and this is due to an increase in the degree of salting of the cutting tool. In this case, the roughness parameter of the treated surfaces will depend on the level of cooling lubricant cleaning in the magnetic sump. An analysis of the results allows us to establish that with a decrease in the concentration of mechanical impurities after cleaning in the magnetic sump (from 1,0 g/l to $0,01 \mathrm{~g} / \mathrm{l}$ ), the parameter of surface roughness of the samples decreases from $R_{a}=0,8 \mu \mathrm{m}$ до $R_{a}=0,2 \mu \mathrm{m}$.

The influence of the purity index of cooling lubricant in the magnetic sump on the roughness of the grinded surface of the samples is shown in Fig. 6 [14].

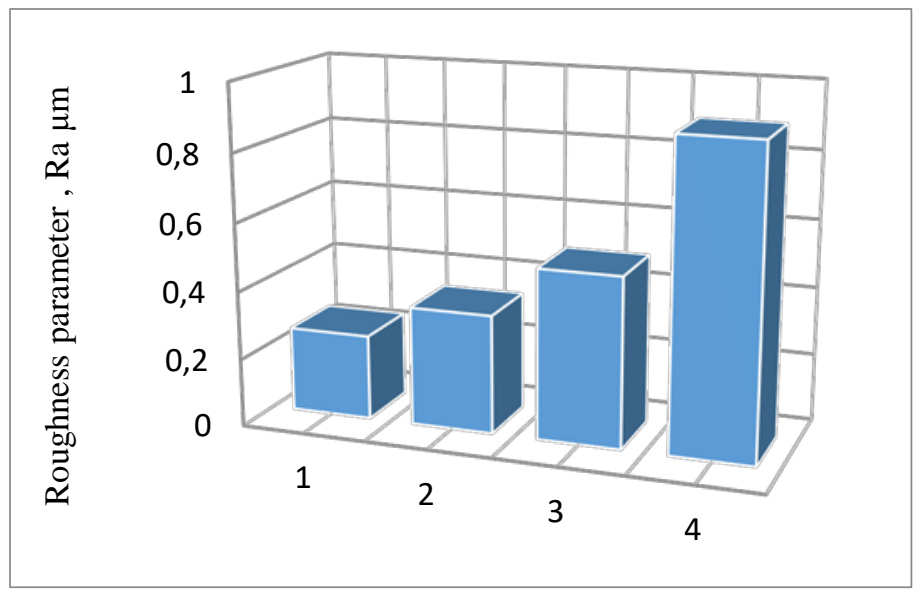

Fig. 6. Dependence of the roughness parameter of the samples on the purity index of the cooling lubricant in the magnetic sump: $1-\delta_{\text {out }}=0,01 \mathrm{~g} / \mathrm{l} ; 2-\delta_{\text {out }}=0,1 \mathrm{~g} / \mathrm{l} ; 3-\delta_{\text {out }}=0,4 \mathrm{~g} / \mathrm{l} ; 4-\delta_{\text {out }}=1,0$ $\mathrm{g} / \mathrm{l}$

The influence of the level of cooling lubricant cleaning in a magnetic sump on the number of grinded shaft journals before dressing of a circle is shown in Fig. 7. 


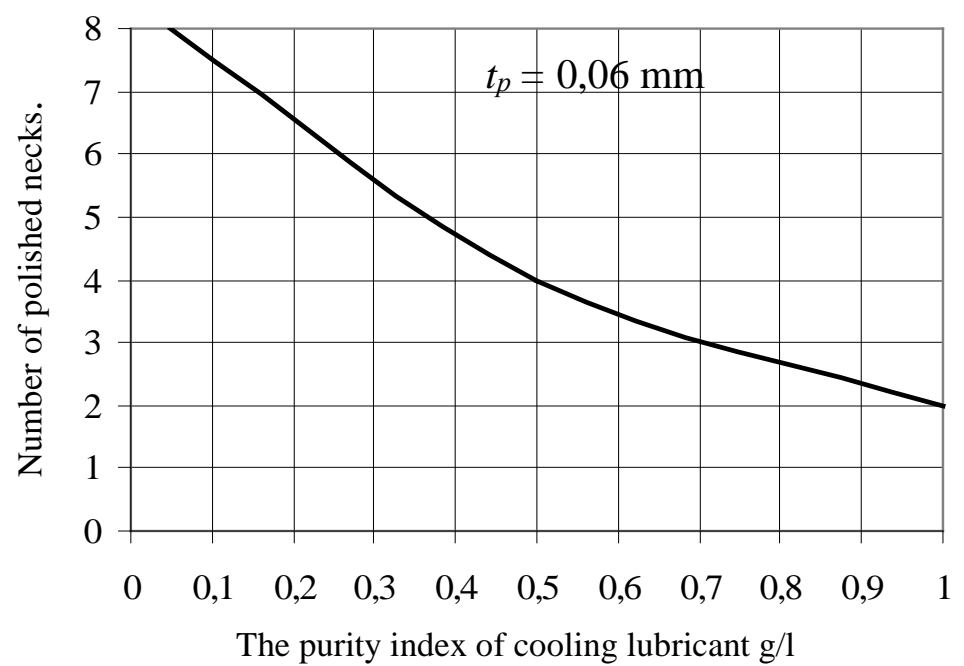

Fig. 7. Influence of cooling lubricant purity in a magnetic sump on the number of grinded shaft journals (before dressing of a circle)

Influence of the level of cooling lubricant cleaning in a magnetic sump on productivity of the grinding process of the crankshaft is shown in Fig. 8 [5].

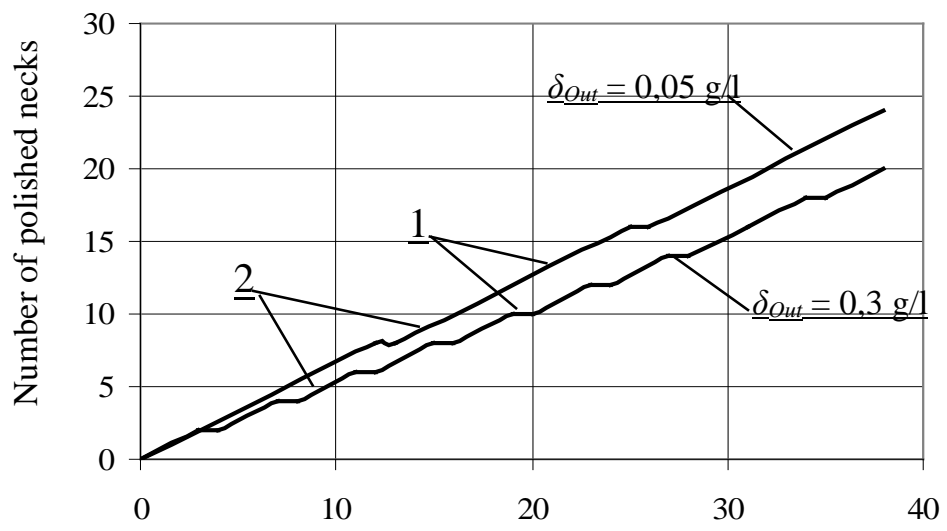

Processing time of grinding, $\mathrm{s}$

Fig. 8. Effect of coolant purity in a magnetic settler on the performance of the grinding process of the crankshaft necks: 1 - machine time; 2 - edit circle

\section{Conclusion}

Analysis of the derived dependencies allows us to conclude that the cleaning of the cooling lubricant in the magnetic sump up to limiting values according to State All-Russian Standard 50558-93, provides an increase in interval of machine time between cleaning the circle up to $70 \%$. The surface roughness of the detail decreases from $50 \%$ to $30 \%$, in this case immediately after cleaning cooling lubricant, which is explained by a decrease in the degree of greasing of the grinding wheel. The most rational surface roughness $\left(R_{a}=0,32 \mu \mathrm{m}\right)$ when processing the crankshaft of ZIL engine using cooling lubricant cleaned in a magnetic sump 
is achieved with the following parameters of the magnetic sump: length of sump, $L=0,65$ $\mathrm{m}$; width of sump, $A=0.12 \mathrm{~m}$, height of sump $h=0,45 \mathrm{~m}$, number of turns $W=2800$; current in the winding $I=23-25 \mathrm{~A}$, power $P=453 \mathrm{~W}$.

\section{References}

1. B. Avdeyev, MATEC Web Conf.: Int. Conf. on Mod. Trends Manuf. Technol. Equip. 129, 06012 (2017)

2. V.A. Zhukov, E.P. Masyutkin, B.A. Avdeyev, IOP Conf. Ser.: Mater. Sci. Eng., 177 (2017)

3. B. A. Avdeyev, V. B. Masyagin, R. R. Dema, O. B. Kalugina IOP Conf. Series: Journal of Physics: Conf. Series, 1050012005 (2018)

4. V.A. Zhukov, A.A. Pulyaev, O.V. Melnik, A.P. Nyrkov, Proceedings of the 2019 IEEE Conference of Russian Young Researchers in Electrical and Electronic Engineering, ElConRus (2019)

5. S. Chernyi, IOP Conf. Ser.: Mater. Sci. Eng., 124 (2017)

6. A.N. Shapovalov, R.R. Dema, S.P. Nefedyev and others, J. Chem. Technol. Metall., 52(5), 1008-1016 (2017)

7. E. Sokolova, MATEC Web Conf.: Int. Conf. on Mod. Trends in Manuf. Technol. and Equip. 12903008 (2017)

8. A.A. Sokolov, A.S. Miroshnikov, E.A. Sokolova, Gornyi Zhurnal, 12, 83 (2016)

9. R.R. Dema, R.N. Amirov, O.B. Kalugina, Lecture Notes in Mechanical Engineering, 9783319956299, 929-937 (2019)

10. S.I. Platov, R.R. Dema, M.V. Kharchenko, R.N. Amirov, 3rd International Scientific and Technical Conference on Scientific and Technical Progress in Ferrous Metallurgy SATPIFM 2017 (2018)

11. A.P. Morgunov, V.B. Masyagin, V.V. Derkach, N.A. Matveev, Journal of Physics: Conference Series, 858, 012019 (2017)

12. Nyrkov, A. Shnurenko, S. Sokolov, S. Chernyi, \& V. Korotkov, Procedia Engineering, 178, (2017).

13. E. E. Aleksandrov, I. A. Kravec, E. N. Lysikov and other, Increase the resource of technical systems through the use of electric and magnetic fields. Monograph (Khar'kov: NTU KhPI 2006)

14. M. I. Shkarupa, V. B. Dudakov, M. M. Lashnev and V. A. Rogov, Refractories and Industrial Ceramics 58(3) (2017) 